\title{
Application of Software Design in Teaching and Learning
}

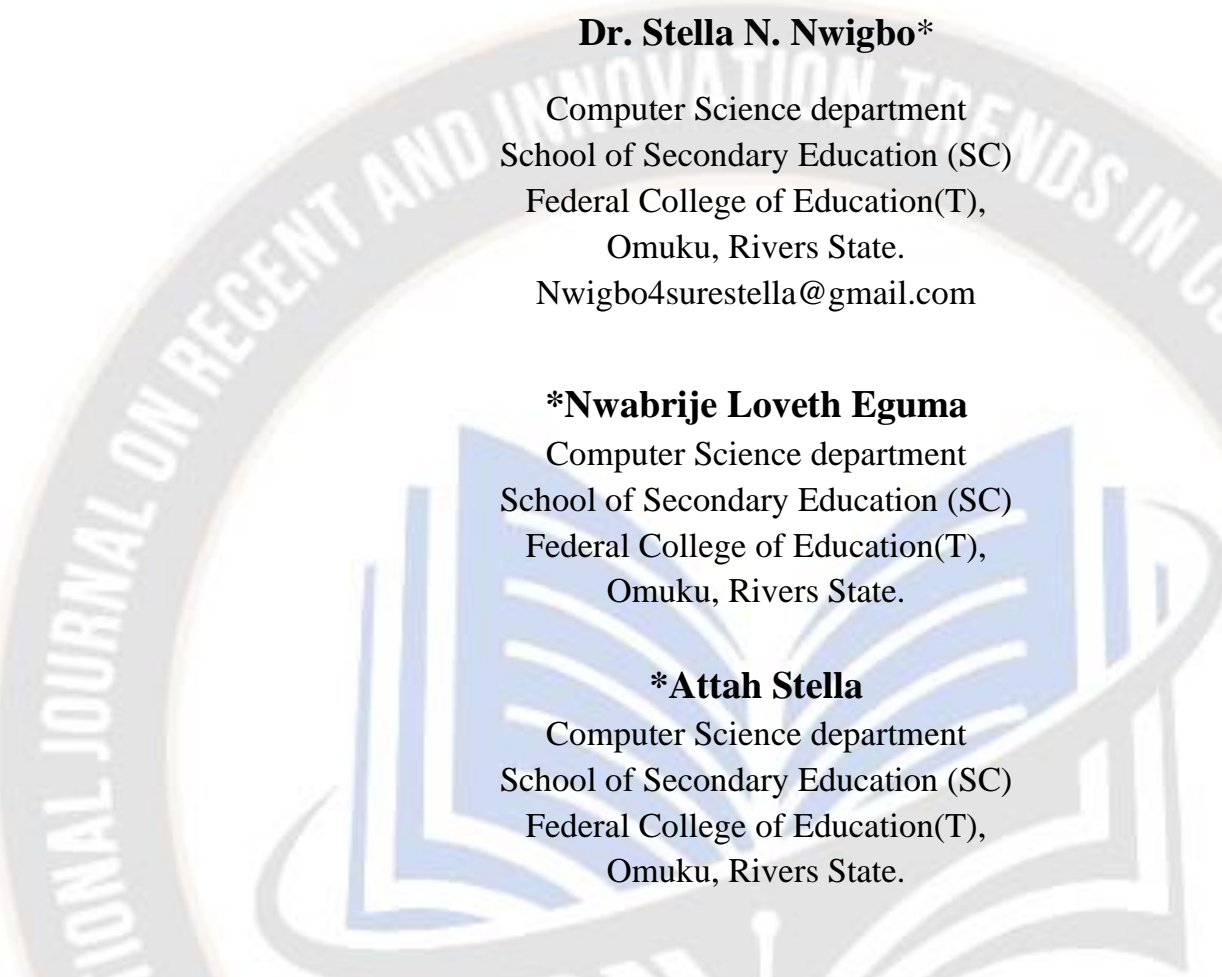

Abstract: The search for solution to the various needs of man and the development of computer technology has resulted in the development of various computer software that provides solutions to these problems. Computer software are designed to address specific challenges. The applications of computer software cuts across the various spheres of life. This paper presents some basic concepts about software design; design pattern, levels and as well discusses some basic application areas of software designs.

Key words - Artifacts functionality, Behavior, Structure, Testability and Maintainability

\subsection{Introduction}

Software is computer term used to describe or identify a variety of computer program that devices embedded with computer technologies work with. They are created from codes or instructions. These codes are sets of instructions that can be converted into a format readable by a computer system. It is a combination of semantics, logic, linguistics, mathematical notations, knowledge representation and system engineering (Zhang and Budgen, 2012).

Javapoint Service (2018), software design involves the transformation of user requirements into forms that are easily implementable using programming languages. It is a complex cognitive process involving participation of people in discussions, exchange of ideas and decision making (Tang et al, 2018). 
In many software designs, aspect such as artifacts functionality, behaviour, structure, testability, maintainability, verifiabilityand usability are some requirements in the design of software. Other factors include completeness, efficiency, flexibility, correctness and consistency. They are otherwise referred to as the principles of software design (Simon, 1973).

One of the most important aspect of software design is design reasoning. There are various reasoning techniques employed in software design. Tang et al (2010) presented some design reasoning techniques applied by most software developers in the design of software. These techniques were grouped into two; inductive reasoning technique and deductive reasoning technique. Whereas inductive reasoning technique involves generalization of specific facts or observations to create theory to be applied to another situation, deductive reasoning technique employs commonly known observations and facts to a logical conclusion for design. Therefore, inductive reasoning technique is exploratory and births new theories while deductive reasoning techniques develops a conclusion from known facts.

Several researchers have described the process of software design as a complex process. To handle this complexity in design, Java developed the following steps to ease design process;

- Problem partitioning: This involves dividing the problem into smaller pieces so as to enable solving each sub-problem separately. Problem portioning creates ease in the understanding by simplifying it.

- Abstraction: This enables a designer to consider a component at an abstract level without bothering about the internal details of the implementation.

- Modularity: This is the division of software into separate modules which enable the designer to address them separately and thereafter, integrate them to obtain a complete functional software.

- Design strategy: The process where the designer decides on the approach to employ in design.

\subsection{Software Design Pattern}

Alexander et al (1977) in their work, presented twenty-three design patterns for software design. They noted that each design pattern tries to describe a unique and reoccurring problem in a particular environment and describes the core of the solution to the particular problem in such a manner that such solution can be used in millions of times without ever doing it same way ever. However, Gamma et al (1995) categorized the 23 patterns into three broad categories; structural, creational and behavioural.

Hussain et al (2017) argued that the choice of a design pattern is hugely affected by the experience of the designer. An experienced designer has higher understanding of design patterns and the nature of problems they solve. However, a novice or a starter may be confused on the required pattern to adopt in design. Also, Zhang \&Budgen (2012) stated that design patterns are useful and valuable to experienced designers than to starters as the former can use them to extend their knowledge schema.

\subsection{Levels of Software Design}

Joshi (2017) categorized software design into three levels as presented and explained briefly below.

- Architectural Design: This is the first level in software design. It is the summarized edition of the system that captures the software as a method with more elements working in synergy to form a system.

- High-level Design: At this level, the concept single entity-multiple component 
is separated into less-abstracted prospect of subsystems. It focuses on how system can be applied as modules and considering the connection and cooperation between the system's modules.

- Detailed Design: This level of design deals with the accomplishment part of the software. It determines the logical structure of all the modules.

\subsection{Application Areas of Software Design}

The area of application of software design are rapidly spread across various spheres of life and still growing including transportation, communications, entertainment, business information processing, health care, manufacturing, utilities, construction, sales, financial services, education, personal computing etc. This section presents examples of software designed for use in above named sectors.

\subsection{Transportation}

Software has increased application in transportation sector ranging from the manufacturing to operations of automobile, aircrafts etc. Some CAD software such as Solidwork, AutoCAD, ANSYS have been employed in the design of automobiles and aircrafts. In the aviation sector, some maintenance, repair and overhaul (MRO) software have been in use. Examples include 2MoRO, OESES, EmpowerMX, ADS, AviiT, AviationSoftware, TRAX, Omega, Aerosoft Systems etc (Ucler\&Gok, 2015).

Software application in automotive system started about four decades ago when a software that controls the engine and the ignition system was introduced (Broy et al, 2007). Currently, the use of software has been developed for various systems of automobiles for its production, operation and maintenance. Software such as the Ramp. 5, Tally. ERP, Marg ERP 9+ software, ACME insight, InvoMax, AutoRepair Cloud,
AutoTracker Plus have been developed for use in the automotive industry.

\subsection{Communication}

A variety of software applications are used in the business process of telecommunication as the telecommunication industry is a software intensive industry. The sales, service provision, charging and billing, service management carried out in the telecommunication business are all regulated by software (Luoma et al, 2010).

\subsection{Entertainment}

Entertainment activities are very important for dynamic living and helps to formulate quality of place identified as one important source of civic satisfaction (Portney, 2013). Entertainment has been observed to promote happiness and wellbeing of citizens, improve quality of life and sense of pride of people, making settlements livable and sustainable and alleviate the pressure of urban living through promotion of social cohesion and reducing social inequality (McCarthy, 2002).

The advancement in technology has helped developed entertainment as software applications have been incorporated into the various channels of entertainment. Software such as Logic Pro, Pro Tools, and Apple Garageband have been developed for music production. Boomplay, MusicMix, GooglePlay and other applications have been in place for playing of music. Spotify, Mixcloud, Pandora and other of similar software have made online streaming of music easy for users. Similarly, there are many other software used in the movie industry. Filmora, Kinemaster and Final Cut Pro are few among many other movie production software just as YouTube, Snagfilm, TubeTv, Crackle and others have been developed for use in the streaming of videos online.

In sports, a plethora of software have been developed and in use. From the management of 
sports to their transmission, tons of software applications have been incorporated for flexibility, ease of management and many other purposes. Some examples include Goalline, Owlwise, PlayyOn and RhinoFit.

\subsection{HealthCare}

According to research 2 guidance (2016), there are over 325,000 health-related software applications directed at patients and health professionals. Software designers are constantly providing new tools for healthcare provisions. Medical diagnoses and many other healthcare activities are currently implemented with the aid of computer technology facilitated by software applications.

A significant development is the introduction of mobile health applications. These applications are mostly designed for use on mobile devices and to provide quick and timely advice on health conditions to their users. They are capable of facilitating the collection and sharing of health data. It has also been observed that the relationship between patients and health care providers is enhanced through the use of mobile health applications (Qudah\&Leutsch, 2019).

The Health Internet-of-Things is another area where software applications have improved healthcare. Stakeholders in healthcare are able to share health information amongst themselves through the aid of software technology. Knowledge is easily transferred amongst health professionalsthrough this technology (Dhanvijay\&Patil, 2019).

\subsection{Manufacturing}

Currently, software have been implemented to automate manufacturing processes from the aspect of design to manufacturing (Vergnano et al, 2017). Computer-Aided Design (CAD) and Computer-Aided Manufacturing (CAM) systems use software to implement designs and manufacturing. In CAM, the software help in converting the design models into information that can be used by machines in manufacturing. The software in use deliver information on how equipment should perform production operations such as cutting, milling and roughing. Also, the software allow the production managers to plan production operations.

Some examples of CAM software include SolidWorks CAM, GibbsCAM, HM, MasterCAM, / PowerMILL, FeatureCAM,RhinoCAM, SprutCAM, ESPRIT, hyperMILL, MachineWorks and SmartCAM.

\subsection{Construction}

Software packages have been integrated into the building or assembly of structures for increased productivity, efficiency and competitiveness with most of these software implemented in design, survey, cost estimation, project management and scheduling (Serror et al, 2008).

Software used in construction industry varies based on the purpose. They could be for prequalification/bidding (iSqFt, Smart BidNet, Viewpoint), project management (Primavera, Microsoft Project, Prolog, PlanGrid), job site data collection (BIM360, Procore, NoteVault) or Design (Revit, AutoCAD, ArchiCAD, Navistocks, MIDAS Civil).

\subsection{Financial Institution}

There has been great acceleration in the level of changes observed in the financial industry with development in technology, internet adoption, mobile penetration in addition to governance policy on cashless society (ePay Summit, 2019).

The advent of software design has improved many banking services such as internet banking, payment system, financial security, data collection and data transfer (Sinkey, 1998). Software design has transformed banking from a primitive 
approach to banking to a modern, easy way with more activities achieved.

\subsection{Education}

The development of computer technology resulted in the design of many software applications that have been employed in the education sector. The use of computer software in education has resulted in many positive impacts such as making teaching more interesting by developing curiosity and motivation in learners, safe teaching and learning time and as well, help improve knowledge of learners. The internet has reshaped learning as many information are easily accessible online. Also, distant education has been made easy as well through internet education (Triantafyllakos et al, 2011).

More so, the education sector has witnessed development of software applications in educational robots and educational games which have helped to facilitate teaching and learning.

\section{Conclusion/ Future Framework}

Basic concepts of software design and its applications have been examined in this work. Software design applications have been shown to have applications in almost every sphere of life. Software applications have made tasks easy as shown in the various sectors discussed in this work.

Software are designed primarily to solve problems and the design varies based on the nature of the problem. This work briefly discussed software design patterns and choice of pattern. It is shown that the choice of design pattern is determined by the designer based on the problem it intends to address.

\section{Reference}

[1]. Simon, H. A. (1973). The structure of ill structured problems. Artificial Intelligence, 4(3/4), $181-201$.

[2]. Tang, A. Aleti, A., Burge, J. \& van Vliet, H. (2010) What makes software design effective. Design Studies, $31(6)$, ***
[3]. Tang, A. Bex, F., Schriek, C. van der Werf, J. M. E. M. (2018) Improving software design reasoning - A reminder card approach.

[4]. Zhang, C. \&Budgen, D. (2012) What do we know about the effectiveness of software design patterns? IEEE Transactionsons on Software Engineering, 38, 12131231.

[5]. Alexander, C. Shikawa, S., Silverstein, M., Jacobson, M., Fiksdahl-King, I., \&Angel, S. A Pattern Language, Oxford University Press, 1977.

[6]. Hussain, S., Keung, J. \& Khan, A. A. (2017) Software design patterns classification and selection using text categorization approach. Applied Soft Computing, 58, $225-244$.

[7]. Gamma, E. Helm, R., Johnson, R., \&Vlissices, J. Design Patterns: Elements of Reusable Object-Oriented Software. Addison-Wesley, Boston, MA, 1995.

[8]. Javapoint (2018) [https://www.javapoint.com/softwareengineering-software-design.]

[9]. Joshi, G. (2017) Different types of software design levels. [https://www.findnerd.com/list/view/differenttypes-of-software-design-levels/13397/]

[10].Munoz, R. G., Shehab, E., Weinitzke, M., Bence, R., Fowler, C. \&Baguley, P. (2015) Towards software performance monitoring: An approach for the Aerospace Industry. In Proceeding of CIRP, Fourth International Conference on Through-Life Engineering Services, 38, $41-46$.

[11].Ulcer, C. \&Gok, O. (2015) Innovating General Aviation MRO's through IT: The Sky Aircraft Management System - SAMS. In 2015 World Conference on Technology Innovation and Entrepreneurship, 1495 1513.

[12].Broy, M., Kruyer, I. H., Prestschner, A., \& Salzmann, C. (2007) Engineering automotive software. Proceedings of IEEE, 95(2), 356 - 373.

[13].Luoma, E., Mazhelis, O. \&Paakkolanvaara, P. (2010) Software-as-a-service in the telecommunication industry: Problems and Opportunities. International Conference of Software Business, 51, $138-150$.

[14].Isakovic, K. (1996) 3D engines list. [http://www.cs.tuberlin.de/\%7Eki/engines.html]

[15].Portney, K. E. Taking sustainable cities seriously: Economic development, the environment and quality of life in American cities. MIT Press, 2013.

[16].McCarthy, C. (2002) Entertainment-led regeneration: The case of Detroit. Cities, 9(2), $105-111$.

[17].Research 2 Guidance (2016) mhealth App Developer Economics.

[18].Dhanvijay, M. M. \&Patil, S. C. (2019) Internet of Things: A survey of enabling technologies in healthcare and its applications. Computer Networks, 153, 113 131.

[19].Qudah, B. \&Luetsch, K. (2019) The influence of mobile health applications on patient-healthcare provider relationships: A systematic, narrative review. ***

[20].Vergano, A., Berselli, G., Pelliciari, M. (2017) Interactiv simulation-base-training tools for manufacturing systems 
operators: an industrial case study. International Journal on Interactive Design and Manufacturing (IJIDeM), 11(4), $785-797$.

[21].Serror, M. H., Inoue, J., Adachi, Y. \&Fujino, Y. (2008) Shared computer-aided structural design model for construction industry (infrastructure). Computer-Aided Design, 40 (7), 778 - 788.

[22].Sinkey, J. F. (1998) Commercial Bank Financial Management Hall.

[23].ePay Summit (2019) GCC payment landscape.

[24]. Available at https://www.epaysummit.com/gccpayments-landscape.

[25]. Trianfyllakos, G., Palaigeorgion, G. \&Tsoukalas, I. A. (2011) Designing educational software with students through collaborative design games: The We!Design\&Play framework. Computers \& Education, 56(1), $227-242$. 